\title{
Padronização do teste imunoalérgico aplicado ao diagnóstico da tuberculose e micobacterioses em suínos (Sus scrofa) experimentalmente sensibilizados com suspensões oleosas de Mycobacterium bovis ou M. avium inativados ${ }^{1}$
}

\author{
Flávia C.S. Oliveira ${ }^{2}$, Sônia R. Pinheiro ${ }^{2}$, Sérgio S. Azevedo ${ }^{3}$, Carolina S.A.B. Santos², \\ Walter Lilenbaum ${ }^{4}$, Francisco R.M. Soto ${ }^{5}$, Eliana Roxo ${ }^{6}$ e Sílvio A. Vasconcellos ${ }^{2 *}$
}

\begin{abstract}
Oliveira F.C.S., Pinheiro S.R., Azevedo S.S., Santos C.S.A.B., Lilenbaum W., Soto F.R.M., Roxo E. \& Vasconcellos S.A. 2014. [Standardization of the immunoallergic skin test applied for the diagnosis of tuberculosis and mycobacterioses in swine (Sus scrofa) experimentally sensitized with oil suspensions of inactivated Mycobacterium bovis or M. avium.] Padronização do teste imunoalérgico aplicado ao diagnóstico da tuberculose e micobacterioses em suínos (Sus scrofa) experimentalmente sensibilizados com suspensões oleosas de Mycobacterium bovis ou M. avium inativados. Pesquisa Veterinária Brasileira 34(2):123-128. Departamento de Medicina Veterinária Preventiva e Saúde Animal, Faculdade de Medicina Veterinária e Zootecnia, Universidade de São Paulo, Av. Prof. Dr. Orlando Marques de Paiva 87, São Paulo, SP 05508-270, Brazil. E-mail: savasco@usp.br

The diagnostic value of the cutaneous allergic response to tuberculin in piglets experimentally sensitized intramuscularly with the oily suspensions of heat inactivated $M$. bovis or M. avium was investigated. Ninety-one animals were used and divided into four groups: groups A and B were formed each with 25 individuals, and groups C and D, with 21 and 20 individuals, respectively, balancing the characteristics of race, ancestry, age and sex. At the age of 30 days, all the animals were submitted to the screening test with the use of $M$. bovis PPD, by the intradermal route at the base of the ear and no reaction was detected. Sixty days after the screening tuberculin test, animals of the group A were injected intramuscularly with $0.5 \mathrm{~mL}$ of oily suspension of $M$. avium D4 strain; animals of the group B received $0.5 \mathrm{~mL}$ of an oily suspension of M. bovis, AN5 strain; group C (control I) received $0.5 \mathrm{~mL}$ of an oily adjuvant; and the individuals of the group D (control II) received $0.5 \mathrm{~mL}$ of saline solution. Following 30 days of sensitization, comparative skin reactions were measured by the variation in skin thickness with a caliper at $0 \mathrm{~h}, 24 \mathrm{~h}, 48 \mathrm{~h}$ an $72 \mathrm{~h}$ after applications of tuberculins. In the comparative test measured at 48 or $72 \mathrm{~h}$, the reaction was considered negative when the difference of the reactions between bovine PPD and avian PPD was less than $6.7 \mathrm{~mm}$; suspected or inconclusive, when the difference stood in the range of 6.7 to $7.5 \mathrm{~mm}$; and positive according to the type of PPD, considering tuberculosis the M. bovis PPD and mycobacteriosis the M. avium PPD, when the difference of the reaction was greater than $7.5 \mathrm{~mm}$.
\end{abstract}

INDEX TERMS: Mycobacterium bovis, Mycobacterium avium, tuberculosis, mycobacteriosis, pigs, tuberculin test, standardization.

\footnotetext{
${ }^{1}$ Recebido em 10 de outubro de 2013.

Aceito para publicação em 4 de janeiro de 2014.

${ }^{2}$ Departamento de Medicina Veterinária Preventiva e Saúde Animal, Faculdade de Medicina Veterinária e Zootecnia (FMVZ), Universidade de São Paulo (USP), Av. Prof. Dr. Orlando Marques de Paiva 87, São Paulo, SP 05508-270, Brasil. *Autor para correspondência: savasco@usp.br

${ }^{3}$ Unidade Acadêmica de Medicina Veterinária, Centro de Saúde e Tecnologia Rural (CSTR), Universidade Federal de Campina Grande (UFCG), Patos, PB 58700-970, Brasil.
}

\footnotetext{
${ }^{4}$ Instituto Biomédico, Centro de Ciências Médicas, Universidade Federal Fluminense (UFF), Rua Prof. Hernani Mello 101, Niterói, RJ 24210-030, Brasil.

${ }^{5}$ Instituto Federal de Educação, Ciência e Tecnologia, Campus São Roque, Rodovia Prefeito Quintino de Lima 2100, São Roque, SP 18136-540, Brasil.

${ }^{6}$ Centro de Pesquisa e Desenvolvimento em Sanidade Animal, Instituto Biológico de São Paulo, Avenida Conselheiro Rodrigues Alves 1252, São Paulo, SP 04014-002, Brasil.
} 
RESUMO.- Foi investigado o valor diagnóstico da resposta alérgica cutânea em leitões experimentalmente sensibilizados, pela via intramuscular, com suspensões oleosas de Mycobacterium bovis ou M. avium inativados pelo calor. Foram utilizados 91 animais, divididos em quatro grupos: grupos A e B, cada um com 25 indivíduos, grupos C e D com 21 e 20 indivíduos respectivamente, balanceando-se as características de raça, linhagem, faixa etária e sexo. Aos 30 dias de idade, todos os animais foram submetidos a uma triagem com a aplicação de tuberculina PPD bovina, pela via intradérmica na base da orelha e não houve qualquer tipo de reação. Decorridos 60 dias do teste tuberculínico de triagem, o grupo A recebeu injeção intramuscular de $0,5 \mathrm{~mL}$ de uma suspensão oleosa de M. avium estirpe D4; o grupo B recebeu $0,5 \mathrm{~mL}$ de uma suspensão oleosa de $M$. bovis estirpe AN5; o grupo $\mathrm{C}$ (controle I), recebeu $0,5 \mathrm{~mL}$ do adjuvante oleoso; e o grupo D (controle II), recebeu 0,5 mL de solução fisiológica. Após 30 dias da sensibilização foi realizada a prova de tuberculinização comparativa com reação medida pela variação da espessura da pele com cutímetro de mola às $0 \mathrm{~h}, 24 \mathrm{~h}, 48 \mathrm{~h}$ e $72 \mathrm{~h}$, após a aplicação das tuberculinas. No teste comparativo, lido às 48 ou 72 horas, a reação foi considerada negativa quando a diferença das reações entre o PPD bovino e o PPD aviário foi menor que 6,7 mm; suspeito ou inconclusivo quando a diferença se situou na faixa de 6,7 a 7,5 mm; e positiva de acordo com o tipo de PPD, considerando-se tuberculose para PPD M. bovis e micobacteriose para PPD M. avium, quando a diferença da reação foi superior a 7,5 $\mathrm{mm}$.

TERMOS DE INDEXAÇ̃̃O: Mycobacterium bovis, Mycobacterium avium, tuberculose, micobacteriose, suínos, teste de tuberculinização, padronização.

\section{INTRODUÇÃO}

Nos últimos anos, o intenso crescimento da suinocultura brasileira transformou suinocultores em empresários rurais e independentemente do tamanho da criação, os desafios e dificuldades têm contribuído para a formação de profissionais capacitados para o gerenciamento das granjas de produção. Por isso, a carne suína reconhecida internacionalmente como segura, excelente para o consumo interno para a exportação, vem ocupando privilegiadas fatias do mercado mundial (Gonçalves \& Palmeira 2006).

Dentre as zoonoses que acometem os suínos, a tuberculose e as micobacterioses são doenças infecciosas crônicas e cosmopolitas, sendo que esses animais podem ser infectados por Mycobacterium tuberculosis, M. bovis, várias espécies do complexo MAIS (M. avium, M. intracellulare e M. scrofulaceum), e ainda por outras micobactérias (Thoen \& Karlson 1986). As micobactérias do complexo MAIS causam lesões granulomatosas nos linfonodos do trato gastrointestinal de suínos, a linfadenite granulomatosa, que leva a sérias perdas no abate desses animais, ocasionando prejuízos tanto para o produtor quanto para a indústria (Brasil 2006, Morés et al. 2007). No ser humano, a infecção pelas micobactérias do complexo MAIS tem importância para os indivíduos com deficiência imunológica. Esta condição situa o suíno como uma importante espécie "sentine- la", reveladora da presença de microrganismos causadores da tuberculose e de micobacterioses em uma determinada região (Acha \& Szyfres 2001).

A tecnificação da suinocultura fez com que a ocorrência da tuberculose por M. tuberculosis em suínos passasse a ser rara, pois o hábito de alimentar suínos com restos de alimentos humanos foi abandonado, contudo a infecção por M. bovis e micobactérias oportunistas ou ambientais persiste e tem sido associada, respectivamente, ao uso de soro de leite e a partidas de cama de maravalha contaminadas (Alfredsen \& Skjerve 1993, Morés et al. 2006).

No Brasil, para uma granja de reprodutores de suínos ser classificada como Granja de Suínos Certificados (GSC), a legislação do Ministério da Agricultura Pecuária e Abastecimento, na Instrução Normativa no 12 de 23 de junho de 1999, estabelece que a granja deva realizar, periodicamente, exames laboratoriais e provas diagnósticas para a peste suína clássica, doença de Aujeszky, brucelose, tuberculose, leptospirose e sarna, bem como para outras doenças, a critério do Departamento de Defesa Animal. No caso da tuberculose, todos os animais devem ser testados com tuberculina PPD mamífera a cada 12 meses. Caso haja positividade é recomendado o emprego da prova comparativa. Contudo, a despeito de constar da legislação, ainda não existe um consenso definitivo sobre a execução e interpretação do teste tuberculínico intradérmico aplicado na espécie suína, particularmente nos rebanhos acometidos por micobacterioses causadas por micobactérias do complexo MAIS, principal responsável por casos de linfadenite tuberculóide em suínos.

Para fins de diagnóstico, o Programa Nacional de Sanidade Suídea (PNSS) recomenda a prova da tuberculinização comparada com tuberculina aviária e bovina inoculadas na dose de 0,05 mg de PPD por via intradérmica (face dorsal da orelha) e a leitura feita com o auxílio de paquímetro ou régua. A leitura deve ser realizada 48 horas após a inoculação das PPDs aviária e bovina. A interpretação do teste é realizada para o rebanho e não para o indivíduo e com base nos experimentos realizados por Morés et al. (2006) em suínos experimentalmente infectados com $M$. avium e M. bovis ficou estabelecido que quando a média do maior diâmetro das reações para o PPD aviário ultrapassar o valor de $0,5 \mathrm{~cm}$ o rebanho está infectado por micobactérias oportunistas, e se o valor médio do diâmetro das reações for igual ou superior a $0,5 \mathrm{~cm}$ para o $\mathrm{PPD}$ bovino, o rebanho está infectado por M. bovis.

A disponibilização de um instrumento de diagnóstico passível de aplicação nos suínos, que permita a diferenciação individual de animais infectados por microrganismos do complexo M. tuberculosis da provocada por micobactérias oportunistas poderá servir como instrumento para o monitoramento e vigilância epidemiológica dos rebanhos, possibilitando a implantação de medidas preventivas e de saneamento (Horsburgh 1991, Sobestiansky et al. 2001). Dessa maneira, o objetivo do presente trabalho foi padronizar um teste imunoalérgico aplicado ao diagnóstico da tuberculose e micobacterioses em suínos experimentalmente sensibilizados com suspensões oleosas de $M$. bovis ou $M$. avium inativados. 


\section{MATERIAL E MÉTODOS}

Foram utilizados 91 leitões sadios, provenientes de uma granja comercial de suínos, localizada no Município de Ibiúna, no Estado de São Paulo, não reagentes ao teste imunoalérgico intradérmico comparado com tuberculina mamífera e aviária, na base da orelha, executado segundo as orientações adotadas no Brasil para a espécie suína (Brasil 2004). Ao nascimento, todos os animais foram identificados com marcação numérica nas orelhas pelo sistema australiano (Maqueda 1999, Lima et al. 2004). Os animais tinham 30 dias de vida no inicio do experimento, pertenciam aos cruzamentos entre às raças Landrace e Large White, apresentavam peso médio de 6 a $7 \mathrm{~kg}$, e foram mantidos no esquema de manejo usual da granja: até os 21 dias de vida os leitões foram mantidos em celas parideiras individuais e micro ambiente com aquecimento e aleitamento materno; após os 21 dias, foram desmamados e encaminhados ao setor de creche em baias coletivas de alvenaria, onde permaneceram até os 45 dias de vida com aquecimento, ração balanceada e água à vontade; após os 45 dias os animais foram destinados à área de engorda onde permaneceram até o momento do abate.

Decorridos 60 dias do teste tuberculínico utilizado como triagem inicial, tempo esperado para que desaparecesse qualquer interferência do antígeno injetado (Tammemagi \& Simmons 1971), os animais foram distribuídos em dois grupos com 25 indivíduos e outros dois grupos, um deles contendo 21 e o outro 20 animais, balanceando-se as características de raça, linhagem, faixa etária e sexo e inoculados via intramuscular na tábua do pescoço com os inóculos padrões e com os respectivos controles (dia experimental zero). 0 grupo A recebeu a injeção intramuscular de $0,5 \mathrm{~mL}$ de uma suspensão oleosa inativada de Myobacterium avium estirpe D4; o grupo B recebeu 0,5 mL de uma suspensão oleosa inativada de $M$. bovis estirpe AN5; o grupo C (controle I), recebeu 0,5 mL do adjuvante oleoso; e o grupo D (controle II) recebeu $0,5 \mathrm{~mL}$ de salina (solução fisiológica). As doses foram aplicadas segundo o protocolo disponibilizado pelo Lanagro/MG.

Decorridos 30 dias da sensibilização, foi realizado o teste comparativo de tuberculina, injetando-se 0,1mL de PPD aviário (tuberculina aviária/0,5mg/mL) e 0,1 mL de PPD bovino (tuberculina bovina $/ 1 \mathrm{mg} / \mathrm{mL}$ ), via intradérmica, na base da orelha, sendo o PPD bovino aplicado na orelha esquerda, e o PPD aviário na orelha direita. Para aplicação das tuberculinas, foram utilizadas seringas e agulhas de insulina, cuidando-se para que, por ocasião da aplicação, houvesse a formação de uma pequena elevação da pele, semelhante a uma lentilha. A espessura da pele foi efetuada com cutímetro de mola no momento imediato anterior à aplicação da tuberculina (momento 0) e decorridas 24, 48 e 72 horas pós-tuberculinização.

A avaliação histológica da reação tuberculínica foi realizada através de biópsias de pele, realizadas 72 horas pós-tuberculinização, colhidas com o emprego de punch descartáveis com $10 \mathrm{~mm}$ de diâmetro, nos locais onde foi constatada reação à tuberculina. Foram examinados cinco animais do grupo A, cinco do grupo B, cinco do grupo $\mathrm{C}$ e cinco do grupo D. 0 controle de pele normal foi obtido por biópsias de pele efetuadas na base da orelha de suínos que não foram submetidos a qualquer tipo de injeção intradérmica. Todos os fragmentos foram fixados em formol a 10\% tamponado em frascos estéreis, e encaminhados para o Laboratório de Histologia do Departamento de Patologia da Faculdade de Medicina Veterinária e Zootecnia da Universidade de São Paulo (VPT/ FMVZ/USP), onde ocorreu a confecção das lâminas com posterior coloração pelo método de Hematoxilina-Eosina (Junqueira \& Junqueira 1983).

Foram calculados os intervalos de confiança de 95\% (IC 95\%) superior e inferior para os diferentes tempos de leitura, por tratamento, das respostas homólogas e heterólogas (Sampaio 1998), utilizando-se o programa estatístico Graphpad Instat. Os grupos analisados foram submetidos ao teste de normalidade de Kolmogorov-Smirnov. Como em todas as comparações pelo menos um grupo apresentou distribuição não normal, foram utilizados testes não paramétricos para as análises. Para a comparação dos momentos de leitura por grupo, foi utilizado o teste de Friedman, e para as comparações entre grupos por momento de leitura foi utilizada a análise de variância não-paramétrica (teste de Kruskal-Wallis), com as comparações post hoc realizadas com o teste de Dunn (Zar 1999). O nível de significância adotado foi de $5 \%$ e os dados foram tabulados e processados com o programa estatístico BioEstat 5.0.

\section{RESULTADOS E DISCUSSÃO}

Os resultados dos testes de tuberculinização comparativo em suínos sensibilizados experimentalmente com $\mathrm{Myco-}$ bacterium avium (grupo A) e $M$. bovis (grupo B) estão apresentados nos Quadros 1 e 2. De acordo com o grupo experimental estabelecido, as reações observadas no teste imunoalérgico intradérmico comparativo em suínos experimentalmente sensibilizados com suspensões oleosas de $M$. bovis ou M. avium, permitiram a classificação das reações em homólogas (PPD empregada produzida com o mesmo tipo de micobactéria utilizada para sensibilizar o animal), heteróloga (PPD empregada produzida com tipo de micobactéria distinto do utilizado para sensibilizar o animal) e reações inespecíficas (observadas em animais

Quadro 1. Valores médios \pm desvios padrão (em milímetros) dos aumentos de espessura da pele de suínos, revelados pelo PPD aviário, aplicado na região dorsal da orelha, segundo os grupos experimentais e os momentos da leitura

\begin{tabular}{ccccc}
\hline \multirow{2}{*}{$\begin{array}{c}\text { Grupos } \\
\text { experimentais }\end{array}$} & \multicolumn{4}{c}{$\begin{array}{c}\text { Momentos da leitura } \\
\text { pós-inoculação }\end{array}$} \\
\cline { 2 - 5 } & 0 hora & 24 horas & 48 horas & 72 horas \\
\hline A & $2,90 \pm 0,42^{\mathrm{a} A}$ & $7,68 \pm 2,30^{\mathrm{a} B}$ & $21,25 \pm 9,98^{\mathrm{a} C}$ & $16,40 \pm 8,5^{\mathrm{BC}}$ \\
B & $2,83 \pm, 049^{\mathrm{a} A}$ & $5,18 \pm 1,93^{\mathrm{Bb}}$ & $7,34 \pm 7,38^{\mathrm{Bb}}$ & $7,34 \pm 6,70^{\mathrm{Bbc}}$ \\
C & $2,66 \pm 0,50^{\mathrm{a} A}$ & $3,27 \pm 0,72^{\mathrm{Cb}}$ & $2,93 \pm 0,50^{\mathrm{Cab}}$ & $2,71 \pm 0,57^{\mathrm{cA}}$ \\
D & $2,97 \pm 0,51^{\mathrm{aA}}$ & $4,24 \pm 1,34 \mathrm{~b}^{\mathrm{cB}}$ & $3,21 \pm 0,65 \mathrm{~b}^{\mathrm{cA}}$ & $3,22 \pm 1,05 \mathrm{~b}^{\mathrm{cA}}$
\end{tabular}

Médias com letras minúsculas diferentes, na mesma coluna, indicam diferença estatística $(\mathrm{p}<0,05)$ entre os grupos dentro de cada momento de leitura. Médias com letras maiúsculas diferentes, na mesma linha, indicam diferença estatística $(\mathrm{p}<0,05)$ entre os momentos de leitura dentro de cada grupo.

Quadro 2. Valores médios \pm desvios padrão (em milímetros) dos aumentos de espessura da pele de suínos, revelados pelo PPD bovino, aplicado na região dorsal da orelha, segundo os grupos experimentais e os momentos da leitura

\begin{tabular}{|c|c|c|c|c|}
\hline \multirow[t]{2}{*}{$\begin{array}{c}\text { Grupos } \\
\text { experimentais }\end{array}$} & \multicolumn{4}{|c|}{$\begin{array}{l}\text { Momentos da leitura } \\
\text { pós-inoculação }\end{array}$} \\
\hline & 0 hora & 24 horas & 48 horas & 72 horas \\
\hline A & $2,71 \pm 0,45^{\underline{\mathrm{a}} \mathrm{cA}}$ & $6,82 \pm 2,41^{\mathrm{aB}}$ & $9,00 \pm 7,12^{\mathrm{a} B}$ & $7,71 \pm 9,12^{\mathrm{a} B}$ \\
\hline B & $2,95 \pm 0,52^{\mathrm{a}-\mathrm{bA}}$ & $7,04 \pm 2,08^{\mathrm{aB}}$ & $14,35 \pm 9,02^{\mathrm{a} B C}$ & $17,34 \pm 8,94^{\mathrm{bc}}$ \\
\hline $\mathrm{C}$ & $2,41 \pm 0,48^{\mathrm{cA}}$ & $3,19 \pm 0,54^{\mathrm{bB}}$ & $3,06 \pm 0,64^{\mathrm{Bb}}$ & $2,90 \pm 0,51^{\mathrm{cAB}}$ \\
\hline D & $2,85 \pm 0,43^{\mathrm{acA}}$ & $3,82 \pm 0,89^{b B}$ & $3,28 \pm 0,62 b^{A B}$ & $3,03 \pm 0,92^{a} \mathrm{cA}$ \\
\hline
\end{tabular}

Médias com letras minúsculas diferentes, na mesma coluna, indicam diferença estatística $(\mathrm{p}<0,05)$ entre os grupos dentro de cada momento de leitura. Médias com letras maiúsculas diferentes, na mesma linha, indicam diferença estatística $(\mathrm{p}<0,05)$ entre os momentos de leitura dentro de cada grupo. 
inoculados com adjuvante oleoso ou com solução salina grupos controle).

As reações observadas com a tuberculina PPD M. bovis (Fig.1), nos animais sensibilizados com a suspensão oleosa de M. avium inativado (grupo A) considerada como heteróloga apresentaram o valor médio de 5,0 mm com IC 95\% variando de 1,41 a $8,6 \mathrm{~mm}$. Já com o mesmo tipo de PPD as reações observadas nos animais sensibilizados com a suspensão oleosa de M. bovis inativado (grupo B), consideradas como homólogas, apresentaram o valor médio de $14,39 \mathrm{~mm}$ e o intervalo de confiança variando de 10,87 a $17,91 \mathrm{~mm}$. No grupo D, a média foi $0,21 \mathrm{~mm}$ e o IC $95 \%$ apresentou os limites de $-0,22$ a $0,64 \mathrm{~mm}$. Constata-se, portanto, inexistência de superposição de valores entre os três intervalos de confiança calculados, indicando que as reações obtidas por tipo isolado de tuberculina foram específicas, não existindo a categoria de animais caracterizados como inconclusivos.

As reações observadas com a tuberculina PPD M. avium (Fig.2), nos animais sensibilizados com a suspensão oleosa de M. bovis inativado (grupo B), considerada como heteróloga, apresentaram valor médio de $4,54 \mathrm{~mm}$ com IC $95 \%$ variando de 1,87 a 7,21mm. Já com o mesmo tipo de PPD as reações observadas nos animais sensibilizados com a suspensão oleosa de M. avium inativado (grupo A), consideradas como homólogas, apresentaram o valor médio de $13,5 \mathrm{~mm}$ e o IC $95 \%$ variando de 10,15 a $16,84 \mathrm{~mm}$, e no grupo D, a média foi $0,26 \mathrm{~mm}$ e o IC $95 \%$ apresentou os limites de $-0,16$ a $0,67 \mathrm{~mm}$. Constata-se, portanto, inexistência de superposição de valores entre os três intervalos de confiança calculados, indicando que as reações obtidas por tipo isolado de tuberculina foram específicas, também não existindo a categoria de animais caracterizados como inconclusivos.

Os valores obtidos no teste comparativo, para os quais foram estabelecidas as diferenças dos aumentos de espessura da pele de suínos, induzidos pelo PPD bovino subtraído do encontrado com o PPD aviário $(\Delta \mathrm{B}-\Delta \mathrm{A})$, no teste intradérmico comparado na região dorsal da orelha, às 72

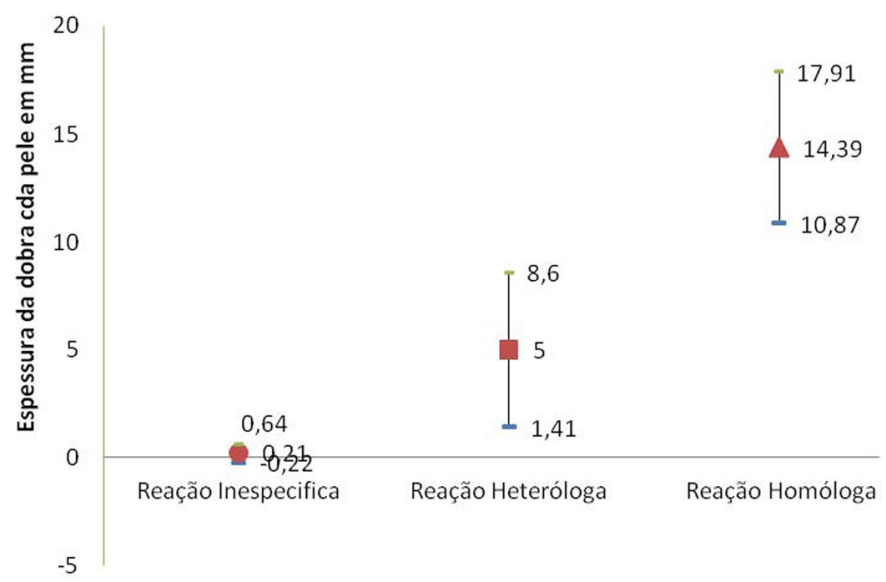

Fig.1. Intervalo de confiança de $95 \%$ da variação da espessura da pele, expressa em milímetros, no ponto de aplicação da tuberculina PPD bovina segundo o grupo experimental: grupo D (reações inespecíficas), grupo A (reações heterólogas) e grupo B (reações homólogas).

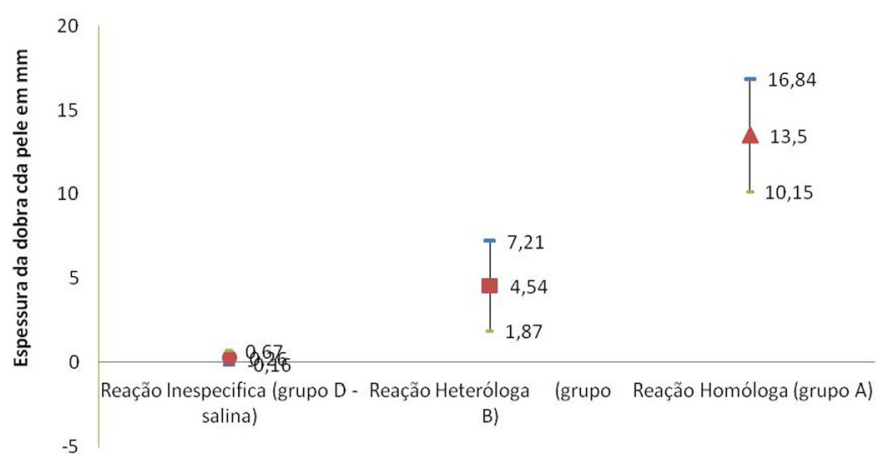

Fig.2. Intervalo de confiança de $95 \%$ da variação da espessura da pele, expressa em milímetros ( $\mathrm{mm}$ ), no ponto de aplicação da tuberculina PPD aviário segundo o grupo experimental: grupo D (reações inespecíficas), grupo A (reações homóloga) e grupo B (reações heteróloga).

horas da aplicação da tuberculina, permitiram o estabelecimento da seguinte chave de interpretação: reação negativa, quando o aumento de espessura da pele for inferior a $6,7 \mathrm{~mm}$ (inespecífica); suspeita, quando o aumento estiver entre 6,7 e 7,5mm (faixa de superposição dos limites inferiores) e positiva, quando o aumento da espessura da pele for maior ou igual a 7,5mm (homóloga). De fato a observação dos intervalos de confiança construídos para os resultados do teste comparativo nos animais dos grupos A e B (Fig.3), revelaram a existência de superposição de valores nos limites inferiores: 6,69 e 7,50 respectivamente para os grupos A e B e, portanto, nesta faixa de valores o resultado do teste comparativo pode apresentar resultado inconclusivo, que não diferencia micobacteriose de tuberculose. Contudo, as reações do teste comparativo com diferença superior a 7,5mm para uma dada tuberculina já podem ser consideradas como capazes de diferenciar infecção por $M$. bovis da provocada por micobacteriose ambiental ou oportunista.

Os valores encontrados no presente trabalho diferiram dos preconizados por Brasil (2004), estabelecidos a partir da investigação efetuada por Morés et al. (2006). Tal diferença poderia ser atribuída às diferenças no delineamento

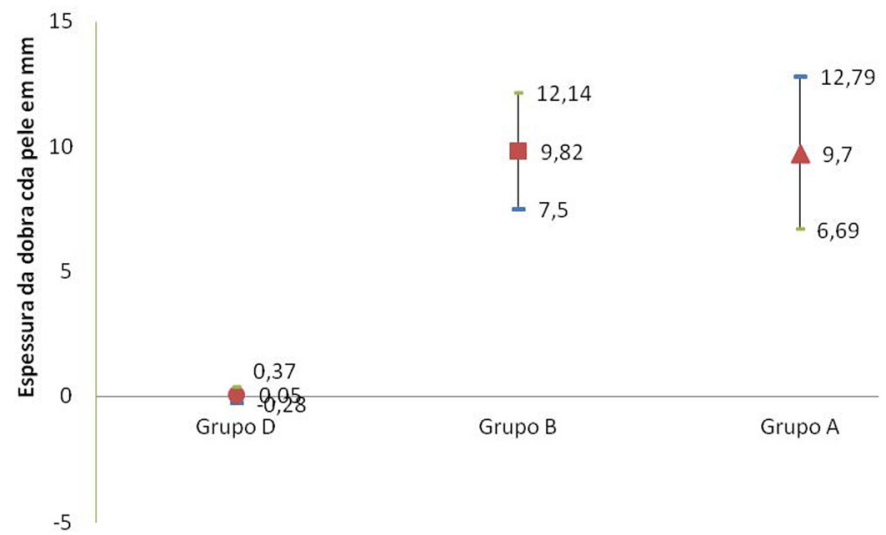

Fig.3. Intervalo de confiança de $95 \%$ da espessura da pele, expressa em milímetros (mm), segundo o grupo (A, B e D) e o tipo de reativo (tuberculina aviária / tuberculina bovina), em relação às diferenças da espessura da pele da tuberculina bovina menos aviária, no teste comparativo. 
experimental adotado por estes autores para o estabelecimento da infecção experimental nos suínos, que empregou as vias oral e intranasal utilizando micobactérias vivas, o que difere do adotado no presente trabalho, que utilizou suspensões oleosas de micobactérias mortas inoculadas pela via intramuscular, o que garantiu que uma quantidade fixa do inóculo fosse realmente apresentada ao sistema imune dos suínos, e isto não pode ser estabelecido no experimento realizado por Morés et al. (2006), pois uma parcela dos microrganismos inoculados em tal experimento poderia ter sido eliminada pelos mecanismos de defesa inata, inespecíficas (Abbas \& Lichtman 2009).

A despeito da importante contribuição oferecida por Silva et al. (2000) e Morés et al. (2006), para o diagnóstico in vivo da tuberculose e micobacteriose suína, o tipo de procedimento adotado para o estabelecimento da infecção experimental pode ter induzido a respostas imunes variáveis, que interferiram na padronização dos critérios de interpretação das respostas obtidas e, consequentemente, na impossibilidade do estabelecimento do diagnóstico individual. Já no presente trabalho tais fontes de variação foram minimizadas, pois a sensibilização dos animais pela via parenteral, com inóculo padronizado possibilitou a indução da resposta imune, com um estímulo antigênico uniforme, em um grupo de animais que possibilitou o cálculo de médias e análise estatística dos resultados obtidos. Dessa forma, foram controladas perdas de inóculo passíveis de ocorrer quando estabelecida a infecção pelas vias oral e intranasal onde, segundo Salazar (2005), os mecanismos inespecíficos de defesa podem interferir com a instalação da infecção.

Na tuberculinização de suínos não pode ser utilizado apenas M. avium, pois esta conduta excluiria o M. bovis, que é o agente patogênico de maior interesse para a saúde pública. Todavia não deve ser somente utilizado $M$. bovis, pois este não faz parte do complexo das micobactérias que, na atualidade, são mais frequentemente encontrados no Brasil em granjas de suínos comerciais. Martins (2001) demonstrou que, na região Sul do Brasil, M. avium é o responsável por $96,4 \%$ das ocorrências de micobacterioses em suínos, enquanto $M$. bovis representa apenas 3,6\% dos isolamentos de linfonodos com lesões granulomatosas.

No presente estudo foi observado que às 72 horas da aplicação do PPD de M. avium as reações intradérmicas dos suínos foram menores que as observadas na leitura efetuada às 48 horas da tuberculinização. Este resultado sugere a possibilidade da realização da leitura do teste já às 48 horas.

0 exame histopatológico de fragmentos de pele de suínos colhidos na área da aplicação da tuberculina, às 72 horas da inoculação, revelou intenso infiltrado inflamatório linfocitário nos animais testados com o PPD homólogo ao tipo de micobactéria utilizada na suspensão oleosa sensibilizante. Estes achados são concordantes com os verificados por Doherty et al. (1996) e Silva et al. (2006).

\section{CONCLUSÃO}

A partir dos resultados encontrados no presente trabalho, propõe-se que, para o teste da tuberculinização comparada em suínos realizado na pele da região dorsal da orelha, com leituras às 48 ou 72 horas da aplicação, a diferença inferior a $6,7 \mathrm{~mm}$ deve ser considerada como negativa; inconclusiva quando na faixa de $6,7-7,5 \mathrm{~mm}$; e positiva quando for superior a 7,5mm, interpretando-se como tuberculose por Mycobacterium bovis, quando o maior valor for o obtido para PPD de $M$. bovis, ou micobacteriose, quando o maior aumento for para o PPD de M. avium.

Agradecimentos.- À Luíz Soto Garcia, proprietário da granja Ouro Preto, localizada no município de Ibiúna-SP, pela liberação dos animais utilizados neste experimento. À Pedro Moacyr Pinto Coelho Mota, Fiscal Federal Agropecuário, Laboratório de Referência Animal do Ministério da Agricultura, Pecuária e Abastecimento (Lara-MAPA/Pedro Leopoldo-MG), pela doação dos inóculos sensibilizantes.

\section{REFERÊNCIAS}

Abbas A.K. \& Lichtman A.H. 2009. Imunologia Básica: funções e distúrbios do sistema imunológico. $3^{\mathrm{a}}$ ed. Elsevier, Rio de Janeiro.

Acha P.N. \& Szyfres B. 2001. Zoonosis y Enfermidades Transmissibles Comunes al Hombre y los Animales. Vol.1. Publicación Científica y Técnica, 580, Organización Panamericana de la Salud, Washington, DC.

Alfredsen S. \& Skjerve E. 1993. An abattoir-based case-control study of risk factors for mycobacteriosis in Norwegian swine. Prev. Vet. Med. 15(4):253-259.

Brasil 2002. Normas para a Certificação de Granjas de Reprodutores Suídeos. Instrução Normativa DAS/MAPA no 19, de 15 fev. 2002. Ministério da Agricultura, Pecuária e Abastecimento, Brasília, DF. 64p.

Brasil 2004. Regulamento Técnico do Programa Nacional de Sanidade Suídea. Instrução Normativa DAS/MAPA no 47, de 18 jun. 2004. Ministério da Agricultura, Pecuária e Abastecimento, Brasília, DF. 64p.

Brasil 2006. Manual Técnico. Programa Nacional de Controle e Erradicação da Brucelose e da Tuberculose Animal (PNCEBT)-MAPA/DAS/DSA, Ministério da Agricultura, Pecuária e Abastecimento. Brasília, DF. 84p.

Doherty M.L., Bassett H.F., Quinn P.J., Davis W.C., Kelly A.P. \& Monaghan M.L. 1996. A sequential study of the bovine tuberculin reaction. Immunol. 87(1):9-14

Gonçalves G.R. \& Palmeira E.M. 2006. Suinocultura Brasileira. Disponível em <http://www.eumed.net/cursecon/ecolat/br/06/rgg.htm> Acessado em 13 set. 2012.

Horsburgh C.R. 1991. M. avium complex infection in the acquired immunodeficiency syndrome. N. Engl. J. Med. 324(19):1332-1338.

Junqueira L.C.U. \& Junqueira L.M.M.S. 1983. Técnicas Básicas de Citologia e Histologia. Livraria e Editora Santos, São Paulo.

Lima J.A.F., Oliveira A.I.G. \& Fialho E.T. 2004. Produção de Suínos. Universidade Federal de Lavras, Lavras, MG.

Maqueda J.J. 1999. Curso para Capacitação em Produção e Sanidade Suína. Consuitec, Campinas, SP.

Martins L.S. 2001. Epidemiologia e controle das micobacterioses em suínos no sul do Brasil: estimativa do impacto econômico e estudo da sazonalidade. Dissertação de Mestrado em Epidemiologia Experimental e Aplicada às Zoonoses, Faculdade de Medicina Veterinária e Zootecnia, Universidade de São Paulo, São Paulo, SP. 51p.

Morés N., Amaral A.L., Ventura L., Silva R.A.M., Silva V.S. \& Barioni Junior W. 2006. Comparação entre métodos de tuberculinização no diagnóstico da infecção por agentes do complexo Mycobacterium avium ou M. bovis em suínos. Arq. Bras. Med. Vet. Zootec. 58(5):1-9.

Morés N., Ventura L., Dutra V., Silva V.S., Barioni Júnior W., Oliveira S.R., Kramer B. \& Ferreira Neto J.S. 2007. Linfadenite granulomatosa em suínos: linfonodos afetados e diagnóstico patológico da infecção causada por agentes do Complexo Mycobacterium avium. Pesq. Vet. Bras. 27(1):13-17.

Salazar F.H.P. 2005. Ocorrência de tuberculose causada por Mycobacte- 
rium bovis em bovinos abatidos em frigoríficos no Estado de Mato Grosso, Brasil. Dissertação de Mestrado em Ciência Animal, Faculdade de Medicina Veterinária e Zootecnia, Universidade Federal de Mato Grosso do Sul, Campo Grande, MS. 68p.

Sampaio I.B.M. 1998. Estatística Aplicada à Experimentação Animal. Fundação de Ensino e Pesquisa em Medicina Veterinária e Zootecnia, Belo Horizonte, MG.

Silva V.S., Morés N., Dutra V.D., Ferreira Neto J.S. \& Saad M.H.F. 2000. Estudo da transmissão horizontal de Mycobacterium avium-intracellulare em suínos. Arq. Bras. Med. Vet. Zootec. 52(6):1-10.

Silva E.G., Pinheiro S.R., Leal M.L.R., Bertagnon H.G., Motta P.M.P.C., Sinhorini I.L., Vasconcellos S.A. \& Benesi F.J. 2006. Teste de tuberculinização em caprinos (Capra hircus) experimentalmente sensibilizados. Ciência Rural 36(3):880-886.

Sobestiansky J., Costa O.A.D., Morés N., Barioni W., Piffer I.A., Guzzo R., Silva V.S. \& Ventura L.V. 2001. Linfadenite granulomatosa em suínos causada por micobactérias atípicas. Comun. Téc. 287, Embrapa Suínos e Aves, Concórdia, SC.

Tammemagi L. \& Simmons G.C. 1971. Pathogenicity of Mycobacterium intracellulare to pigs. Aust. Vet. J. 47(7):337-339.

Thoen C.O. \& Karlson A.G. 1986. Tuberculosis, p.712-729. In: Dunne H.W., Leman A.D., Strow B., Glock R.D., Mengeling W.L., Penny R.H.C. \& Scholl E. (Eds), Diseases of Swine. $6^{\text {th }}$ ed. Iowa State University Press, Ames.

Zar J.H. 1999. Biostatistical Analysis. $4^{\text {th }}$ ed. Prentice Hall, Upper Saddle River. 